1

2

3

4

5

6

7

8

\title{
Relating spinal injury-induced neuropathic pain and spontaneous afferent activity to sleep and respiratory dysfunction
}

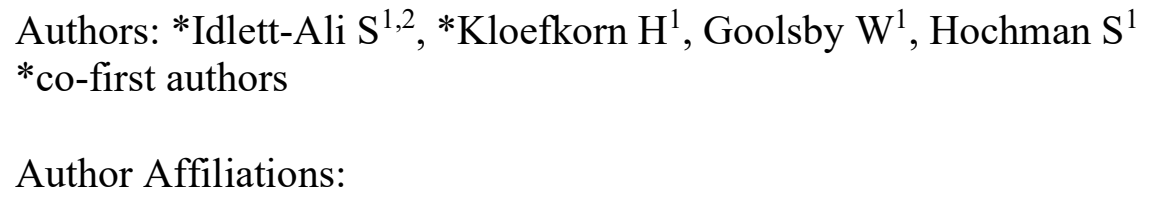

1. Wallace H. Coulter Department of Biomedical Engineering, Georgia Institute of Technology, Atlanta, GA, USA

2. Department of Chemical, Biological, and Environmental Engineering, Oregon State University, Corvallis, OR, USA

3. Department of Physiology, School of Medicine, Emory University, Atlanta, GA, USA

Corresponding Author's full address and current email:

Heidi Kloefkorn

$105 \mathrm{SW} 26^{\text {th }} \mathrm{St}$

Department of Chemical, Biological, and Environmental Engineering

Corvallis, OR

97331

heidi.kloefkorn@oregonstate.edu

Running title: Relating SA afferents to SCI dysfunction

Keywords: spinal cord injury, neuropathic pain, spontaneous afferents, behavioral assays 


\section{ABSTRACT}

42 Spinal cord injury (SCI) can induce dysfunction in a multitude of neural circuits including those

43 that lead to impaired sleep, respiratory dysfunction and neuropathic pain. We used a lower thoracic

44 rodent contusion SCI model - known to develop mechanosensory stimulus hypersensitivity, and

45 spontaneous activity in primary afferents that associates neuropathic pain - and paired this with

46 new approaches that enabled chronic capture of three state sleep and respiration to characterize

47 dysfunction and assess possible interrelations. Noncontact electric field sensors were embedded

48 into home cages for noninvasive capture in naturally behaving mice of the temporal evolution of

49 sleep and respiration changes for 6 weeks after SCI. Hindlimb mechanosensitivity was assessed

50 weekly, and terminal experiments measured primary afferent spontaneous activity in situ from

51 intact lumbar dorsal root ganglia (DRG). We observed that SCI led to increased spontaneous

52 primary afferent activity (both firing rate and the number of spontaneously active DRGs) that

53 correlated with reduced hindpaw mechanical sensitivity, increased respiratory rate variability, and

54 increased sleep fragmentation. This is the first study to measure and link sleep dysfunction and

55 variability in respiratory rate in a SCI model of neuropathic pain, and thereby provide broader

56 insight into the magnitude of overall stress burden initiated by neural circuit dysfunction after SCI. 


\section{BODY OF TEXT}

\section{INTRODUCTION}

Neuropathic pain can develop following peripheral or central nervous system injury, resulting from trauma or disease ${ }^{1}$. Persistent and stimulus-independent neuropathic pain (i.e. spontaneous pain) develops in more than half of spinal cord injury (SCI) patients ${ }^{2}$, yet there remains limited understanding of its underlying mechanisms ${ }^{3}$ and few effective therapies for this debilitating condition.

Many studies explore neurobiological mechanisms and treatment for spontaneous pain using experimental assessment tools optimized for stimulus-evoked allodynia and hyperalgesia ${ }^{4-10}$. Clinically, pain is commonly scored through questionnaires which can be highly subjective and variable 11,12. Preclinically, most modalities of pain are inferred through changes in evoked mechanical or thermal sensory sensitivity, regardless of whether the pain being explored has stimulus-evoked features ${ }^{13,14}$.

Stimulus-evoked assessment methods are considered the semi-quantitative gold standard but their assessment limitations and misuse have, in part, supported arguments that spontaneous pain may represent evoked allodynia and hyperalgesia resulting from unrecognized stimuli ${ }^{3}$. However, there is increasing evidence that spontaneous primary afferent activity represents one initiation site and chronic driver of maladaptive sensory processing, consistent with spontaneous pain perception ${ }^{6}$,

7, 15-17. Spontaneous activity in both A and C fibers has also been observed in many neuropathic pain conditions ${ }^{7,15,18,19}$. In SCI, this peripheral hypersensitivity can predict the development of central pain ${ }^{20,21}$. Key studies in rodent contusion SCI models reveal potential links between stimulus-evoked hypersensitivity (hyperalgesia/allodynia) and the incidence of spontaneous firing in cell bodies of primary afferents below the level of injury $6,16,22,23$. These studies assessed spontaneous activity from C fiber dorsal root ganglia (DRG) neurons following DRG dissociation which may induce hyperexcitability, including increased spontaneous firing that mirrors the effects seen after chronic compression of DRGs ${ }^{24}$. These limitations highlight the need to study intact DRGs to determine the presence of abnormal spontaneous DRG activity after SCI.

To better understand and measure stimulus independent spontaneous pain, non-evoked assessment methods have been developed. For example, the conditioned place preference test ${ }^{25-27}$ and grimace scale ${ }^{28}$ have been shown to differentiate animals with neuropathic pain from those without.

88 Potential compounds of these tests include efficacy of analgesics, animal training, and experimenter interactions ${ }^{14,29}$. There is a need for additional indices of ongoing spontaneous pain during natural behavior with one possibility being emergent changes in other physiological phenomenon. Alternatively, home-cage wheel running has been shown to identify spontaneous migraine pain ${ }^{30}$, however is inappropriate for animal models with confounding motor dysfunction 93 such as SCI.

Impaired sleep is a significant, though often overlooked, component of suffering after SCI with meaningful impacts on life quality ${ }^{31}$. Though SCI patients commonly experience reduced sleep quality ${ }^{32-35}$, research on cause-effect relations between impaired sleep after SCI and additional important co-morbid conditions is limited. While it is well established that sleep disruption and chronic pain are bi-directionally and negatively reinforcing ${ }^{34-36}$, their association in SCI has not been detailed clinically or in preclinical animal models. This is at least partly due to the difficulty 
101 introduce extra surgery, immune response, stress, and altered home cage environments that may 102 complicate behavioral changes and pain after SCI.

103 Similarly, while there are several studies describing respiratory-related sleep dysfunction caused 104 by cervical SCI ${ }^{34,37,38}$, including sleep apnea ${ }^{39}$, preclinical work aimed at understanding whether 105 alterations in respiration associated with SCI -induced neuropathic pain are limited. Using lower 106 thoracic SCI models that do not overtly impair respiratory neural circuits, studies in a restraint 107 cylinder showed respiratory rate increases after thoracic contusion or hemisection injury that 108 associate with the emergence of allodynia ${ }^{40,41}$. This important observation needs to be reproduced 109 during natural behavior, which have relied on whole-body plethysmography ${ }^{42}$ that has limited its 110 applications in SCI animal models ${ }^{43}$.

111 To better quantify spontaneous pain and address limitations of existing pain assays, we explore 112 novel physio-behavioral indices after SCI. In the thoracic contusion SCI model of neuropathic 113 pain, we examine metrics associated with sleep architecture and respiratory function during natural behavior in the animal's home cage using non-invasive electric field sensors ${ }^{42,44}$. We also compare these behavioral outcomes to established mechanical sensitivity testing, along with terminal experiment observations of spontaneous activity in whole DRGs, below the level of injury.

117 A greater understanding of this work reveals a potential link of the pathological sensory state after 118 SCI, with quantitative metrics of sleep and respiration abnormalities. Exploitation of this link could provide a broader perspective into understanding overall stress burden initiated by neural circuit dysfunction after SCI. 


\section{METHODS}

123 Animals

124 All procedures were approved by the Emory University Institutional Animal Care and Use Committee. Adult C57/B16 mice $(\mathrm{n}=20$, female) were pair housed in home cages under standard 12:12 hour light-dark cycles with ad libitum food and water.

\section{Animal surgeries}

128 SCI was induced via moderate contusion of the T10 spinal cord segment ${ }^{45}$. Fourteen adult mice 129 (day 205) were prepared for aseptic surgery and deeply anesthetized with 2-3\% isoflurane. A midline skin incision was made to dissect the muscle and fascia prior to exposing the spinal cord at T8-T10 via a dorsal laminectomy. In six animals (Sham Group, $n=6$ ), wounds were closed at this point. In the eight remaining animals (SCI Group $n=8$ ), a moderate contusion SCI was made dorsally at T9/10 with the Infinite Horizon impactor (50 kdynes, IH-400, Precision Systems and Instrumentations) prior wound closure. All mice received post-operative pain relief $(2 \mathrm{mg} / \mathrm{kg}$ meloxicam), antibiotics ( $2.5 \mathrm{mg} / \mathrm{kg}$ Baytril), supplemental hydration ( $0.5 \mathrm{ml}$ sterile saline daily), and bladder expression twice daily until each animal voided independently. One animal was excluded from these data due to meeting endpoint criteria. Six additional mice served as naïve controls (Naïve, $\mathrm{n}=6$ ).

\section{Physio-behavioral monitoring}

Home cages $\left(32 \times 18 \times 14 \mathrm{~cm}\right.$, Super Mouse ${ }^{\mathrm{TM}}$ microisolator), described previously ${ }^{42,}{ }^{44}$, were instrumented with electric field (EF) sensors (Plessey Semiconductors, PS25251, $1 \mathrm{~cm}^{2},+/-5 \mathrm{~V}, 1$ $\mathrm{kHz}$ sampling) able to passively translate disruptions of the local electric field caused by movement into a voltage trace. EF sensors have been validated against respective gold standard techniques to reliably quantify resting respiration, sleep-wake staging (including differentiating rapid eye movement sleep, REM, from Non-REM sleep), and animal motor activity noninvasively from outside the animals' home cage ${ }^{42,44}$.

Animals were acclimated to their instrumented home cages for several days prior to recording (Figure 1A). The dividing insert was only present during physio-behavioral recordings; cagemates shared the full home cage at all other times. Three baseline recordings were collected prior to surgery (baseline). For six weeks post-surgery, recordings were collected twice weekly at night (6pm-6am) for SCI animals and once weekly for sham and naïve animals.

\section{Sleep analysis}

Sleep was analyzed from the EF sensor home cage recordings. As described previously ${ }^{44,46}$, wake, rapid eye movement (REM) sleep, and non-REM sleep were quantified through changes in animal movement and respiration (Figure 1B). Weekly averages were calculated for total sleep time (TST), percent time spent in each arousal state, average sleep bout duration (SBD), average REM sleep event duration, REM sleep latency, percentage of sleep bouts containing REM sleep, sleep fragmentation index (SFI: total awakenings per hour of sleep), and micro arousal index (MAI: total micro arousals $<60$ seconds per hour of sleep).

\section{Respiration analysis}

Respiration was detected during non-REM sleep and quiet wake by the EF sensors (Figure 1C). Respiratory rate (RR) is the frequency at which the animal breathes and RR variability (RRV) is the standard deviation of the instantaneous frequencies for each breath during a non-REM event. 
Weekly values for RR and RRV were calculated using a custom MATLAB script containing 0.1 $\mathrm{Hz} \mathrm{HP}$ and $10 \mathrm{~Hz} \mathrm{LP} 4^{\text {th }}$ order Butterworth filters.

\section{Mechanical sensitivity}

Mechanical sensitivity was measured using Chaplan's up-down protocol to calculate 50\% paw withdrawal threshold (PWT) ${ }^{47}$. 50\% PWT was assessed three times prior to surgery (baseline) and once weekly afterwards for 6 weeks on the same day as overnight physio-behavioral recordings were taken. Single-animal values were obtained by averaging the $50 \%$ PWT for both hind paws.

\section{Isolation of multisegmental DRGs}

178 An isolated spinal cord mouse preparation ${ }^{48}$ was extended to include DRG from segments C8 through S1. Dorsal and ventral roots were cut to isolate the DRGs and prepared as previously described ${ }^{48}$. All recordings were undertaken at room temperature.

\section{Extracellular DRG recordings}

Dura and pia were removed around the DRG and glass suction electrodes $(200-250 \mu \mathrm{m}$ tip diameter) were positioned on lumbar (L1-L6) DRGs to record spontaneous afferent population activity for a minimum of 25 seconds $(50 \mathrm{kHz}$, Digidata 1322A 16 Bit DAQ, Molecular Devices, U.S.A., Figure 2). Signals were amplified (5000x) and filtered (LP $3 \mathrm{kHz}$ at recording, $\mathrm{LP} 5 \mathrm{~Hz}$ post-hoc). Spontaneously active (SA) and silent DRG spike counts were measured with Clampfit software (v 10.7 Molecular Devices). DRGs were considered SA (SA DRGs) with >1 spike within 25 secs. DRG firing frequency was determined by spike frequency within 5 secs bins. Due to the nonuniform sampling of DRGs across animals, a weighted SA DRG incidence value was calculated given as the ratio of recorded lumbar DRGs of the 12 is the total possible per animal (\#/12).

\section{Statistical analysis} determined using 2-Way ANOVA, with a post hoc Sidak's multiple comparison's test $(\alpha=0.05)$. Two-tailed Mann-Whitney tests were used to analyze DRG firing frequencies and weighted incidences of SA DRGs. Residual changes from baseline in each animal were quantified for 50\% PWT, sleep metrics, and RRV at postoperative week 6 then correlated with post-mortem electrophysiology using Pearson correlation analysis. All statistical analyses were performed using Statistica (Tibco Software Inc. Palo Alto, California) and, unless otherwise stated, all data are presented as mean \pm standard deviation. 


\section{RESULTS}

SCI results in chronic hindpaw hypersensitivity

Mechanical sensitivity was assessed to confirm the injury caused mechanical hypersensitivity in agreement with previous findings ${ }^{49}$. SCI animals developed persistent heightened mechanical sensitivity beginning at week 2 relative to Naïve and Sham groups and SCI baseline (Figure 3 and Table 1).

\section{Respiration becomes more erratic after SCI}

The average resting RR did not show significant differences between treatment groups or across time (Figure 4 and Table 1). At baseline, all treatment groups exhibited a consistent respiratory rate variability (RRV) averaging $0.21 \pm 0.02 \mathrm{~Hz}$. However, RRV increased by week 2 for the SCI group relative to SCI baseline and increased relative to Naïve and Sham groups at all time periods after two weeks.

\section{Sleep becomes fragmented after SCI}

Total sleep time was not significantly different between treatments or across time (Figure 5 and Table1). Beginning at week 2, SCI animals developed progressive fragmented sleep as measured by decreased sleep bout duration, increased SFI, and increased MAI relative to Naïve and Sham groups and SCI baseline. In SCI animals, REM sleep duration, REM latency, and the percentage of sleep bouts containing REM sleep decreased at later timepoints relative to Naïve and Sham groups and SCI baseline. Specifically, changes in REM sleep developed later than both mechanical sensitivity and initial sleep fragmentation.

\section{Greater spontaneous nerve activity emerges after $S C I$}

To assess below-level spontaneous primary afferent activity, spontaneous nerve population activity in L1-L6 DRGs was recorded bilaterally from SCI and Sham animals 6 weeks after injury. Compared to Sham $(0.6 \pm 0.3 \mathrm{~Hz}), \mathrm{SCI}$ animals exhibited greater spontaneous activity $(2.5 \pm 1.2$ $\mathrm{Hz}$ ), as characterized by the maximal DRG firing frequency $(\mathrm{p}<0.005$, Figure 6). The weighted incidence of SA DRGs in the SCI population $(29.8 \pm 16.5 \%)$ was significantly greater than Sham $(12.5 \pm 4.6 \%, p<0.5$, Figure 6).

\section{Spontaneous Hyperexcitability Correlates with Changes in Evoked Hyperexcitability,} Respiration, and Sleep Changes after $S C I$

Correlative relationships between behavioral changes and spontaneous afferent activity were identified (Figure 7). Though direct comparisons between behavior and electrophysiology yield no clear correlations, residualized behavior measures between postoperative week 6 and baseline (i.e. the change measured at week 6 from each animal's respective baseline) reveal significant relationships: the change in mechanical sensitivity $(50 \% \mathrm{PWT})$ negatively correlates with the weighted incidence of SA DRGs, the change in SFI positively correlates with the weighted incidence of SA DRGs, and the change in RRV positively correlates with DRG firing frequency. These findings link sleep and respiratory features to changes in spontaneous afferent activity and neuropathic pain after SCI. 


\section{DISCUSSION}

This work links spontaneous afferent activity, behavioral mechanosensitivity measures consistent with neuropathic pain to measures of sleep and respiration in naturally behaving mice. While peripheral hyperexcitability has been previously observed in SCI models of neuropathic pain, here we found correlations between spontaneous lumbar DRG activity (incidence and firing frequency) and metrics of sleep and respiratory dysfunction - complications frequently observed in SCI patients. The findings suggest that in addition to examining primary afferent activity, non-contact recording of sleep and respiratory features may serve as a useful tool for identifying the emergence of persistent spontaneous neuropathic pain in animal models of SCI. Below we discuss results of SCI -induced changes in sleep, respiration and sensory hyperexcitability changes in separate sections prior to providing our overall perspective.

\section{Sleep changes after SCI}

Currently there is very limited preclinical animal studies devoted to assessing sleep changes after

SCI. Only one study looked at and observed sleep dysfunction independent of respiration ${ }^{50}$. This study was undertaken in rat and follow changes for 15 days after complete lower thoracic spinal tansection and observed a transient reduction in sleep efficiency during the light cycle.

The technological advance of the EF sensors has enabled noninvasive quantification of sleep changes in rodents after SCI ${ }^{44}$. Though the time spent asleep during the 12-hour dark cycle (6pm6am) was unchanged, SCI animals had more fragmented and lower quality sleep relative to their sham and naïve counterparts. There were meaningful reductions in average sleep bout duration (SBD), REM sleep duration, REM sleep latency, and the percentage of sleep bouts containing REM sleep. Sleep fragmentation index (SFI) representing total number of awakenings/hr of sleep, and micro arousal index (MAI) representing total number of $<60$ seconds micro arousals/hr of sleep were increased after SCI. The increased brief arousals and reduced duration of sleep bouts caused compensatory increased number of sleep events after SCI, presumably reflecting experienced increased sleep pressure.

275 Since REM sleep occurs at the end of a sleep cycle, it is intuitive to link these observed reductions in REM sleep to reduced SBD associated with observed increased sleep fragmentation and micro arousals. If sleep events are shortened and without observed REM sleep, it is possible the animal did not achieve normal transitions for restorative sleep. Factors responsible for sleep compromise may include increased pain pathway recruitment of sympathetic drive, given known correspondence with mechanical hypersensitivity and in other studies showing evidence of ongoing spontaneous pain ${ }^{6,51}$. Regardless of mechanism, sleep dysfunction would contribute to overall stress burden after SCI and, through actions on common stress axis pathways contribute to overall disease burden in SCI.

\section{Respiration changes after SCI}

While cervical SCI clearly impair respiration due to direct injury to respiratory neural circuits that impact sleep ${ }^{52-54}$, there is little consideration on whether SCI -induced neural dysfunction in other areas may interact with an impact respiration. Nonetheless, recent work has shown that, when recorded in restraint cylinders, respiratory rate increases after thoracic contusion or hemisection

290 injury that may precede to correspond with the emergence of allodynia ${ }^{40,41}$. In comparison, and 
recordings of respiratory rate in naturally behaving mice, we observed no change in average respiration rate but with increases respiratory rate variability (RRV). These changes also emerged coincident with measures of mechanical hypersensitivity.

Neural basis of neuropathic pain after SCI

In SCI animals, we showed a correlation between the change in paw withdrawal threshold (PWT) and the and dorsal ganglia (DRG) afferent firing incidence, where a greater reduction in PWT was observed in animals with a higher incidence of spontaneously active DRGs. DRG firing frequency and incidence were not correlated, suggesting they may capture disparate aspects of ongoing pain or an alternate non-pain encoding sensory signaling pathway (e.g. metabolic status, baroreceptors, hypoxia). The incidence of spontaneously active DRGs may determine the size/number of dermatomal regions impacted by neuropathic pain - where a larger incidence could be associated with a larger region impacted. Firing frequency may encode the intensity of pain experienced by a single region which could explain the link between PWT and DRG incidence as the rodent hindpaw is innervated by axons with cell bodies in L3-L6 DRGs ${ }^{55}$. Broader hyperexcitability of these lumbar primary afferents may contribute to greater hindpaw sensitivity.

Spontaneous primary afferent activity has been identified as a driver of chronic pain in clinical ${ }^{56-}$ 58 and preclinical 6, 7, 18, 19,22 studies. Key studies conducted in rodent contusion models of SCI revealed potential links between stimulus-evoked hypersensitivity and spontaneous firing of primary afferents below the level of injury $6,16,22,23$. Here, we recorded activity from intact DRGs across multiple vertebral segments to confirm development of increased spontaneous afferent activity after SCI. Though our recording technique did not permit resolution to identify the class of primary afferent nerves within the signal population, there is evidence suggesting spontaneous nerve activity after SCI includes signals from A and C fibers which have been associated with neuropathic pain including in the T10 contusion SCI animal model 6, 7, 15, 16, 18, 19, 22, 58, 59 .

\section{CONCLUSIONS}

We show that a commonly used SCI animal model of neuropathic pain is also associated with coemergent sleep dysfunction and increased respiratory rate variability, thereby providing a broader understanding of the magnitude of overall stress burden initiated by neural circuit dysfunction after SCI. Further studies are warranted to better understand the nature of their interactions be they cause-effect relations or independent parallel injury-induced phenomenon on distinct pathways impacted by SCI as such insights may inform implementation of therapeutic control strategies after SCI.

\section{ACKNOWLEDGMENTS}

We thank Mallika Halder for assisting with the animal surgeries; Sandra Garraway and Karmarcha Martin for training us in the development of the T10 contusion model and permitting the use of the Infinite Horizon impactor.

\section{RESEARCH CONTRIBUTIONS}


334 Animal surgeries were performed by Shaquia Idlett-Ali and Mallika Halder. Tissue isolation, 335 electrophysiology experimentation, and analysis was performed by Shaquia Idlett-Ali. Behavioral 336 testing and analysis was conducted by Heidi Kloefkorn. Shaquia Idlett-Ali, Heidi Kloefkorn and 337 Shawn Hochman were involved in preparation of the manuscript.

AUTHOR DISCLOSURE STATEMENT

340 No competing financial interests exist.

\section{FUNDING STATEMENT}

343 Research supported by the Craig H Neilsen Foundation and the Paralyzed Veterans of America 344 Research Foundation (S.H.). S.I. supported by National Science Foundation Graduate Research 345 Fellowship Grant DGE-1650044 and the Alfred P. Sloan Foundation. H.K supported by NIH 346 fellowship (5k12-Gm000680). 
Figure 1

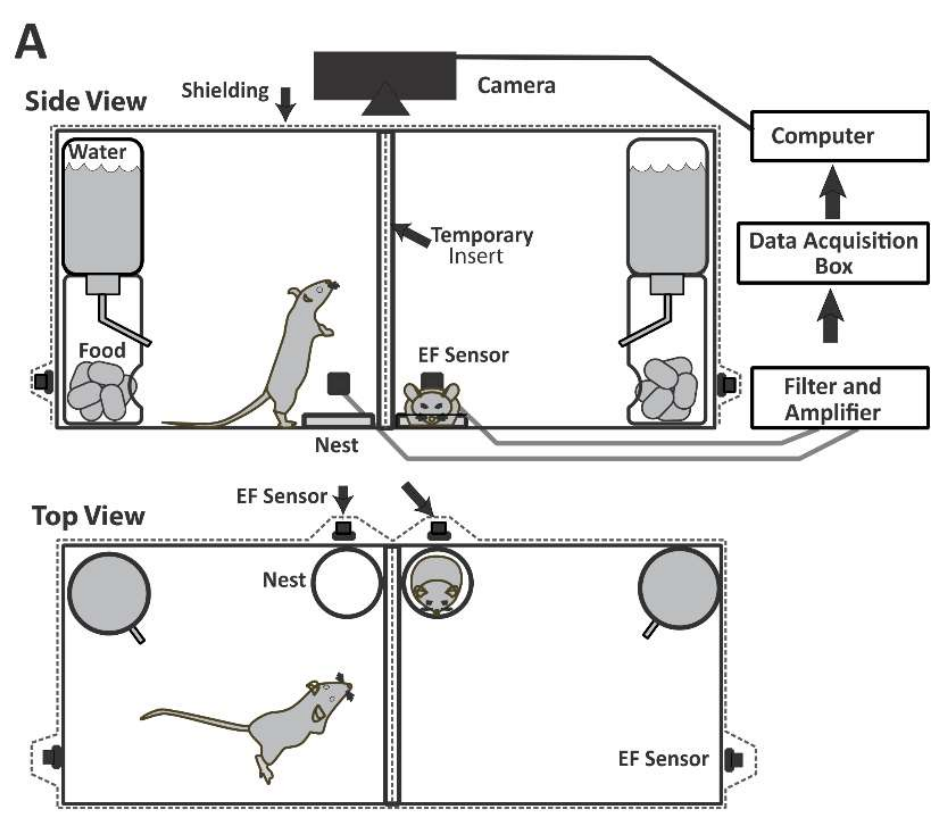

B

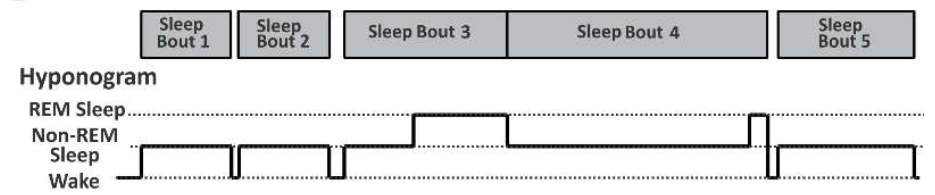

Spectrogram

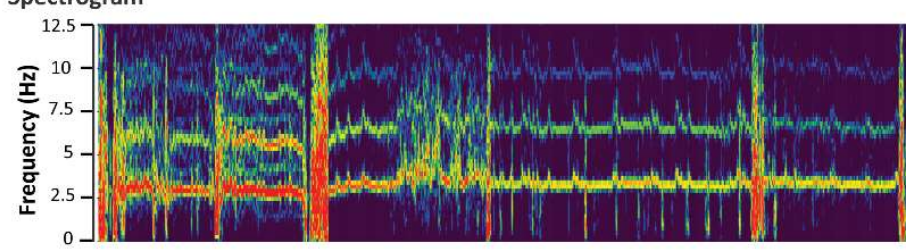

Raw EF Sensor Voltage Trace

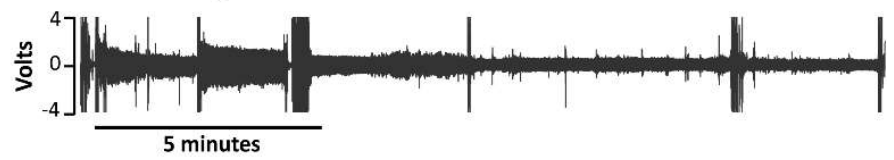

C

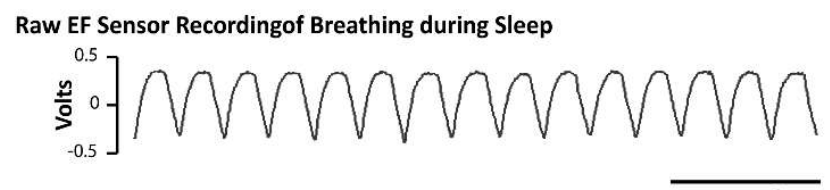

\section{Figure 1: Methodology for non-invasive monitoring of animal sleep and respiration. A)}

Home cage instrumentation for electric field sensing. A total of 4 individual EF sensor were used per cage, with 2 dedicated to each animal. B) Representative spectrogram (top panel) and overlaid hypnogram (white trace) obtained from electric field sensor recording (bottom panel) sensor. 
Figure 2

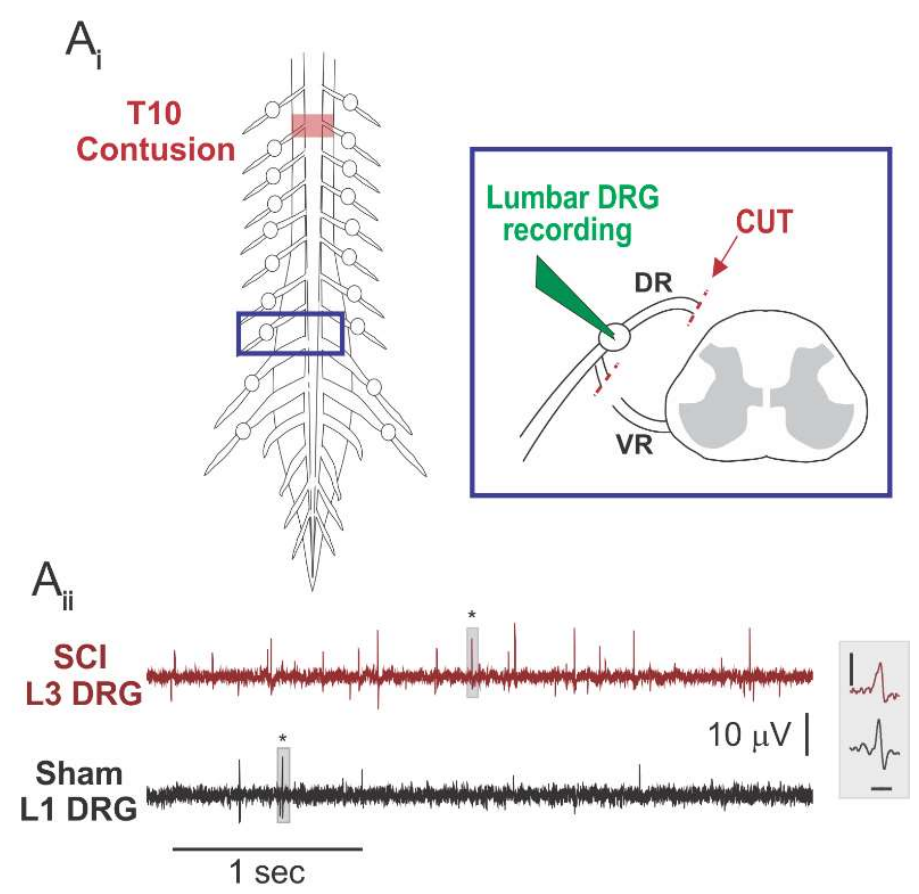

Figure 2: Methodology for electrophysiology for isolated DRG preparation.

Ai) A schematic of the isolated DRG preparation. Following exposure of the spinal cord and peripheral aspects of primary afferents (DRG, dorsal root, spinal nerve) from C8-S1 segments, the dorsal roots (DRs) and ventral roots (VRs) were cut at each segment, leaving only a section of the DRs with the attached DRGs and the surrounding ribs and musculature (not shown). Aii) Example recordings of spontaneous activity in the lumbar (L) L3 DRG from the SCI (red) and DRGs from 2 different animals. Single units marked with * are expanded in the inset (scale bars are $2 \mathrm{~ms}$ and $10 \mu \mathrm{V})$. 


\section{Figure 3}

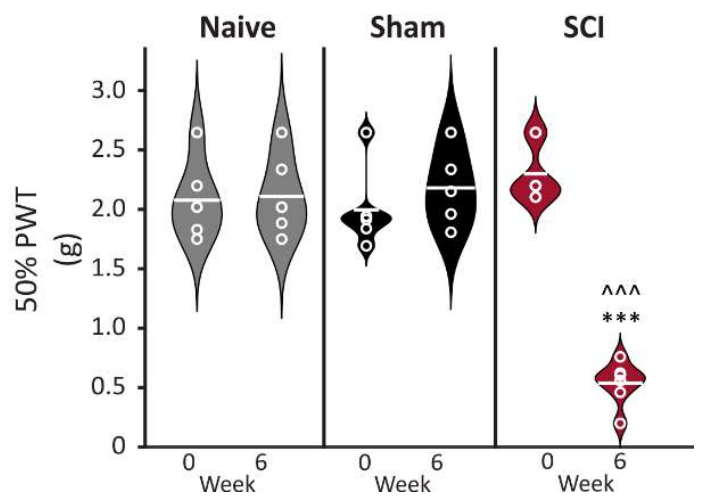

Figure 3: Mechanical Sensitivity. Graph is probability density violin plot of animal averages (circles) with group averages (black and white horizontal lines). Mechanical sensitivity (50\% PWT) is heightened after SCI. The p-values are: SCI vs Sham at week 6- ${ }^{*} \mathrm{p}<0.05, * * \mathrm{p}<0.005$, *** $\mathrm{p}<0.0005$; baseline vs week 6 in the SCI group- ${ }^{\wedge \wedge \wedge} \mathrm{p}<0.0001$. 
Figure 4

A

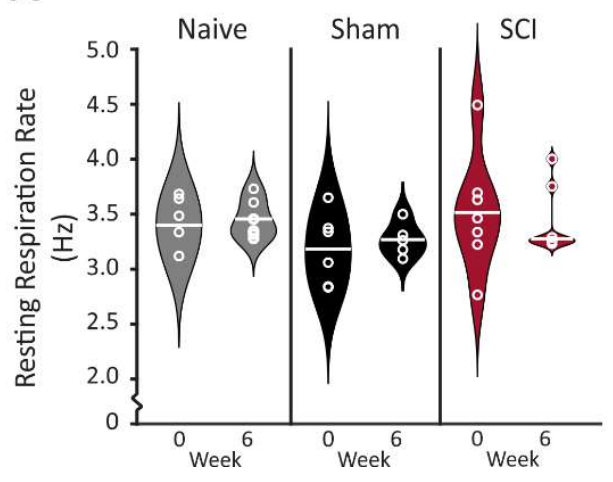

B

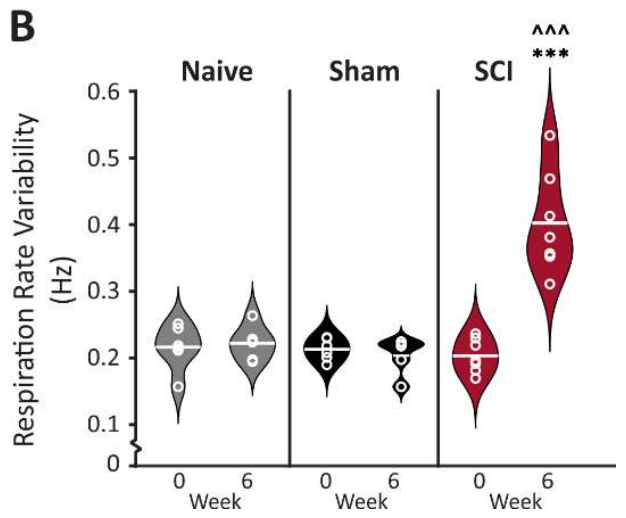

Figure 4: Respiration Changes After SCI. Graphs are probability density violin plots of animal averages (circles) with group averages (black and white horizontal lines). A) Average respiratory rate appears unaffected by SCI, while B) respiratory rate variability differs significantly when comparing SCI vs Sham at postoperative week 6; and baseline vs week 6 in the SCI group. The pvalues are: SCI vs Sham at week $6-{ }^{*} \mathrm{p}<0.05, * * \mathrm{p}<0.005, * * * \mathrm{p}<0.0005$; baseline vs week 6 in the SCI group- ${ }^{\wedge \wedge \wedge} \mathrm{p}<0.0001$. 
Figure 5

A

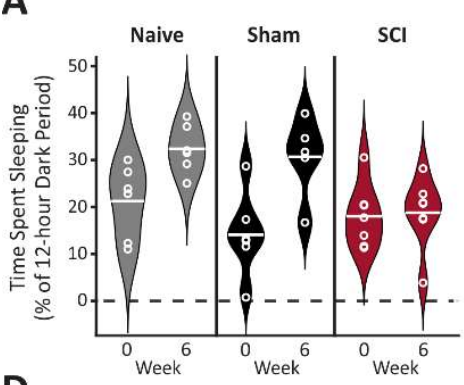

\section{D}

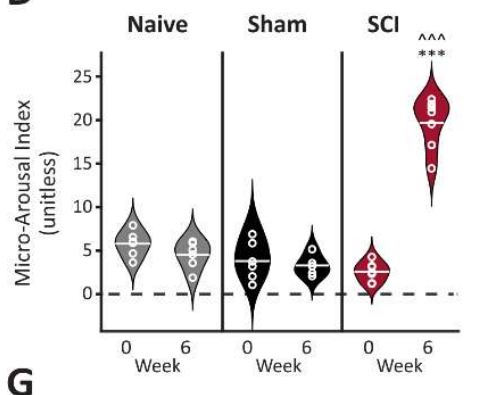

G

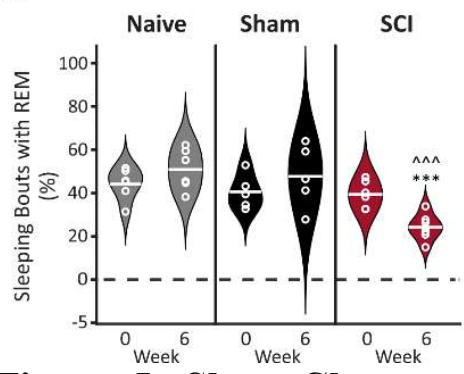

B

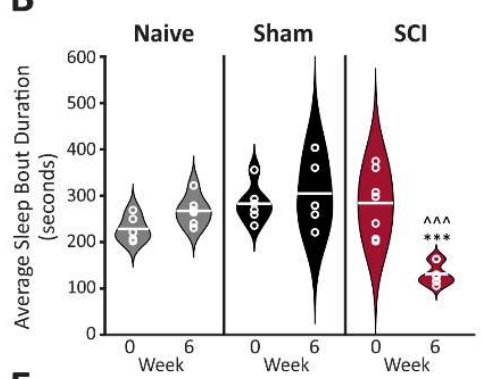

E

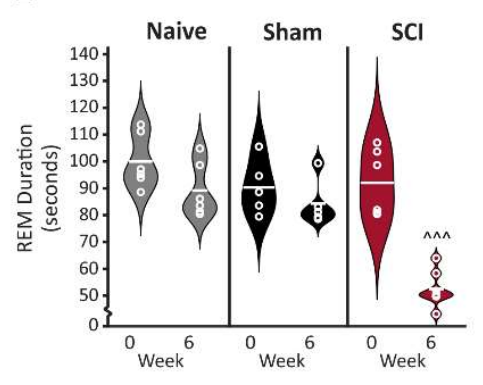

C

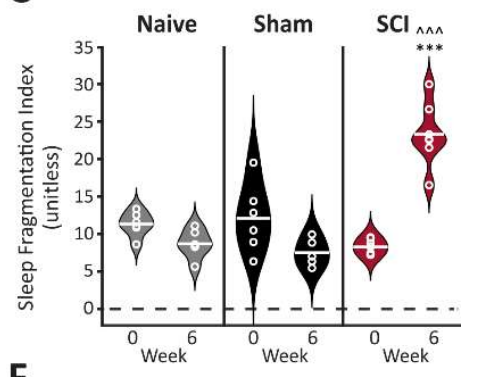

F

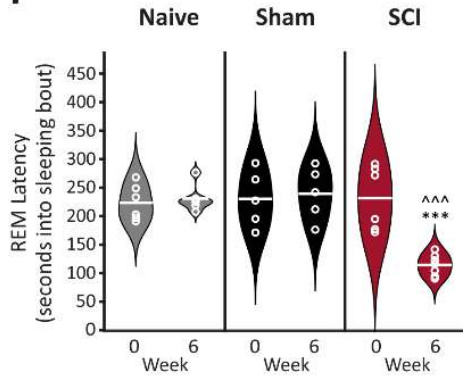

Figure 5: Sleep Changes After SCI. Graphs are probability density violin plots of animal averages (circles) with group averages (black and white horizontal lines). A) While time spent sleeping was unaffected by SCI, B) average sleep bout duration, C) SFI, and D) MAI during a 12hour dark cycle were significantly different when comparing SCI vs Sham at postoperative week 6; and baseline vs week 6 in the SCI group. E) REM sleep duration, F) REM sleep latency, and G) the percentage of sleep bouts containing REM sleep were all reduced in SCI animals at postoperative week 6 relative to their baseline and sham animals at postoperative week 6 (except for REM sleep duration) suggesting premature arousal to wake, an increase in sleep pressure, and an overall reduction in sleep quality. The p-values are: SCI vs Sham at week 6- ${ }^{*} p<0.05$, ** $\mathrm{p}<0.005,{ }^{* * *} \mathrm{p}<0.0005$; baseline vs week 6 in the SCI group- ${ }^{\wedge \wedge \wedge} \mathrm{p}<0.0001$. 
Figure 6
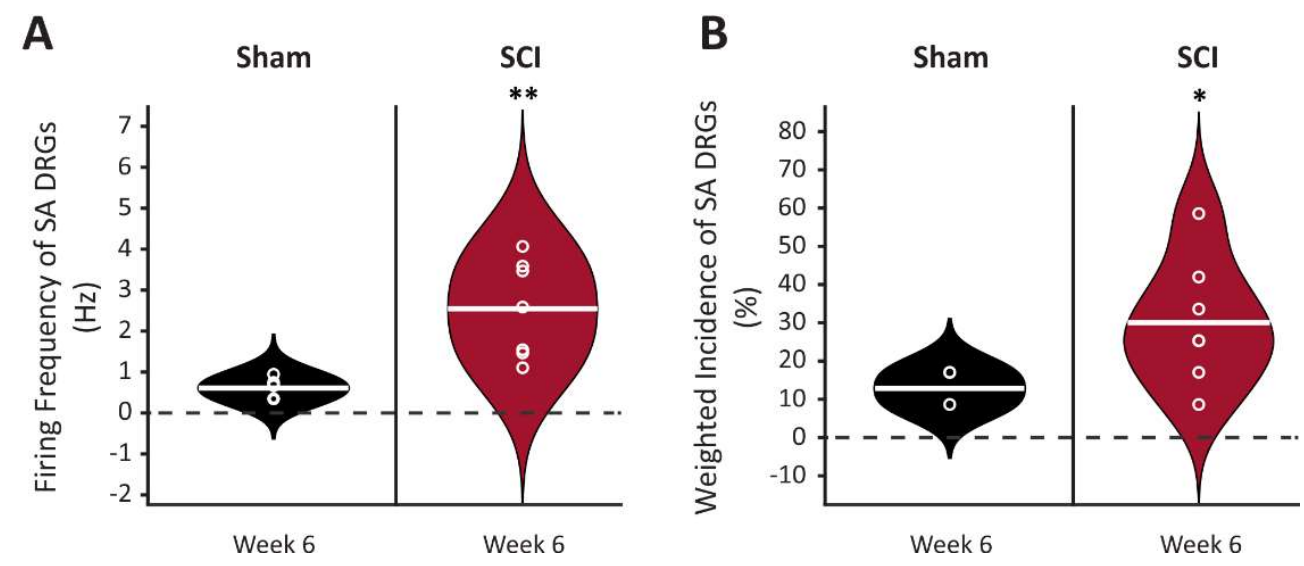

Figure 6: Spontaneous Afferent Firing. Graphs are probability density violin plots of animal averages (circles) with group averages (black and white horizontal lines). A) The maximal observed firing frequency and B) weighted incidence of SA lumbar DRGs in the SCI group are significantly greater than that of the Sham group. SCI $(n=7)$, Sham $(n=5)$. The p-values are: SCI vs Sham at week 6- ${ }^{*} \mathrm{p}<0.05, * * \mathrm{p}<0.005, * * * \mathrm{p}<0.0005$; baseline vs week 6 in the SCI group414 
Figure 7

A

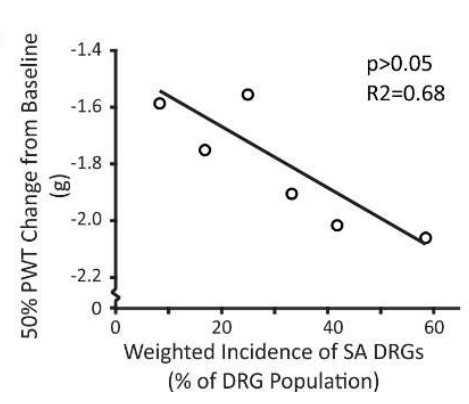

B

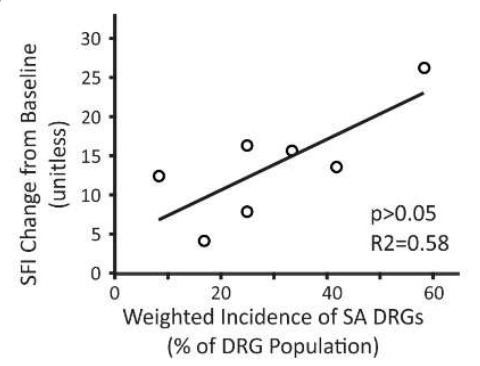

C

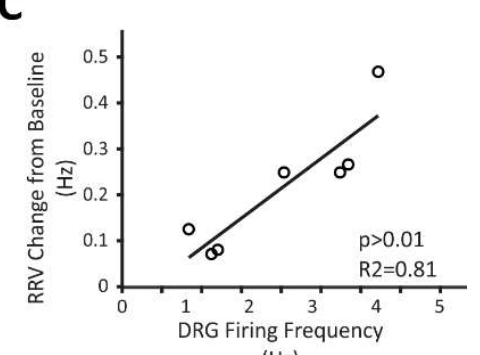

$(\mathrm{Hz})$

Figure 7: Spontaneous hyperexcitability metrics correlate with changes in evoked hyperexcitability, respiration, and sleep after SCI. A) Incidence of SA DRGs in the SCI group has a negative correlation with the change in 50\% PWT threshold (compared to baseline), measured at postoperative week 6. B) In the SCI group, the change in the SFI, which captures the number of sleep disruptions per hour, at week 6 (compared to baseline) has a positive correlation with the incidence of below-injury-level SA DRGs. C) Maximal firing frequency has a positive correlation with the change in respiration rate variability (compared to baseline) at week 6 . Linear associations between metrics were determined by Pearson correlation and regression analysis. Each data point represents a single animal. 
bioRxiv preprint doi: https://doi.org/10.1101/2021.11.15.468636; this version posted November 16, 2021. The copyright holder for this preprint

(which was not certified by peer review) is the author/funder, who has granted bioRxiv a license to display the preprint in perpetuity. It is made available under aCC-BY-NC 4.0 International license.

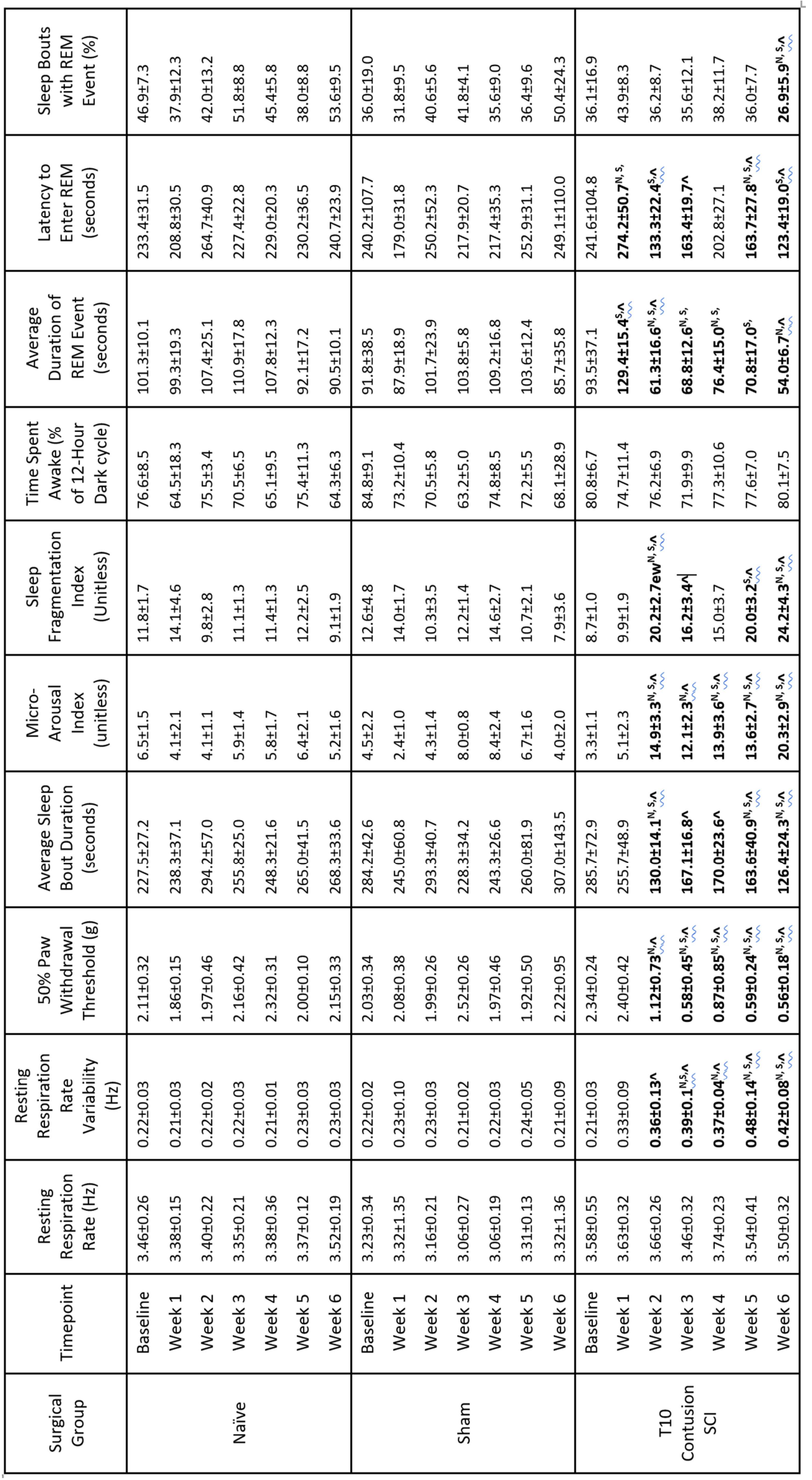


Table 1: Values are shown as mean \pm STD with statistically significant values bolded. ${ }^{\mathbf{N}}$ : 430 Difference from Naïve at respective timepoint $(\mathrm{p}<0.05)$. : Difference from Sham at respective 431 timepoint $(\mathrm{p}<0.05) .^{\wedge}$ : Difference from baseline of respective group $(\mathrm{p}<0.05)$. Resting respiration 432 rate (RR) and percentage of time spent awake were not affected by SCI. By 6 weeks post-injury, 433 SCI animal mechanical sensitivity (50\% PWT), sleep fragmentation indices, and respiration rate 434 variability (RRV) were significantly different from SCI baseline and 6 week Naïve and Sham 435 group values. Specifically, RRV, 50\% PWT, sleep bout duration, sleep fragmentation index (SFI), 436 and micro-arousal index (MAI) showed progressive changes starting at week 2 post-injury, while 437 changes in REM sleep did not solidify until week 6 post-injury. 
1. Kramer, J.L.K., Minhas, N.K., Jutzeler, C.R., Erskine, E.L.K.S., Liu, L.J.W. and Ramer, M.S. (2017). Neuropathic pain following traumatic spinal cord injury: Models, measurement, and mechanisms. Journal of neuroscience research 95, 1295-1306. following spinal cord injury: A systematic review and meta-analysis. European Journal of Pain 21, 29-44. 3. Bennett, G.J. (2012). What is spontaneous pain and who has it? The Journal of Pain 13, 921929. activity of rat dorsal horn cells in spinal segments of sciatic projection following transection of sciatic nerve or of corresponding dorsal roots. Neuroscience 94, 217-228. Excitability in Large Somatosensory Neurons and Its Plastic Changes in Pathological Pain States. Int J Mol Sci 19. Walters, E.T. (2010). Chronic spontaneous activity generated in the somata of primary nociceptors is associated with pain-related behavior after spinal cord injury. J Neurosci. 30, 14870-14882. doi: 14810.11523/JNEUROSCI.12428-14810.12010.

7. Kajander, K.C., Wakisaka, S. and Bennett, G.J. (1992). Spontaneous discharge originates in the dorsal root ganglion at the onset of a painful peripheral neuropathy in the rat. Neuroscience letters 138, 225-228. Xiong, L. (2013). A feed-forward spinal cord glycinergic neural circuit gates mechanical allodynia. The Journal of clinical investigation 123, 4050-4062. 9. Tan, A.M., Chang, Y.-W., Zhao, P., Hains, B.C. and Waxman, S.G. (2011). Rac1-regulated dendritic spine remodeling contributes to neuropathic pain after peripheral nerve injury. Experimental neurology 232, 222-233. 10. Liu, F.-Y., Qu, X.-X., Cai, J., Wang, F.-T., Xing, G.-G. and Wan, Y. (2011). Electrophysiological properties of spinal wide dynamic range neurons in neuropathic pain rats following spinal nerve ligation. Neuroscience bulletin 27, 1-8. 11. Hu, L. and lannetti, G.D. (2019). Neural indicators of perceptual variability of pain across species. Proceedings of the National Academy of Sciences 116, 1782-1791. 12. Koyama, T., McHaffie, J.G., Laurienti, P.J. and Coghill, R.C. (2005). The subjective experience of pain: where expectations become reality. Proceedings of the National Academy of Sciences 102, 12950-12955. 13. Mogil, J.S. (2009). Animal models of pain: progress and challenges. Nature reviews. Neuroscience 10, 283-294.

14. Tappe-Theodor, A., King, T. and Morgan, M.M. (2019). Pros and Cons of Clinically Relevant Methods to Assess Pain in Rodents. Neuroscience \& Biobehavioral Reviews 100, 335-343. 15. Liu, X., Eschenfelder, S., Blenk, K.-H., Jänig, W. and Häbler, H.-J. (2000). Spontaneous activity of axotomized afferent neurons after $L 5$ spinal nerve injury in rats. PAIN ${ }^{\circledR} 84,309-318$. 16. Yang, Q., Wu, Z., Hadden, J.K., Odem, M.A., Zuo, Y., Crook, R.J., Frost, J.A. and Walters, E.T. (2014). Persistent pain after spinal cord injury is maintained by primary afferent activity. The Journal of neuroscience : the official journal of the Society for Neuroscience 34, 10765-10769. 
17. Xie, W., Strong, J.A., Meij, J.T., Zhang, J.M. and Yu, L. (2005). Neuropathic pain: early spontaneous afferent activity is the trigger. Pain 116, 243-256. 18. Song, Y., Li, H.M., Xie, R.G., Yue, Z.F., Song, X.J., Hu, S.J. and Xing, J.L. (2012). Evoked bursting in injured Abeta dorsal root ganglion neurons: a mechanism underlying tactile allodynia. Pain 153, 657-665. 19. Ma, C., Shu, Y., Zheng, Z., Chen, Y., Yao, H., Greenquist, K.W., White, F.A. and LaMotte, R.H. (2003). Similar electrophysiological changes in axotomized and neighboring intact dorsal root ganglion neurons. Journal of neurophysiology 89, 1588-1602. sensory changes following spinal cord injury: predictive properties and implications on the mechanism of central pain. Brain : a journal of neurology 135, 418-430. and Werhagen, L. (2014). Phenotypes and predictors of pain following traumatic spinal cord injury: a prospective study. The journal of pain : official journal of the American Pain Society 15, 40-48. Dysregulation of Kv3.4 channels in dorsal root ganglia following spinal cord injury. The Journal of neuroscience : the official journal of the Society for Neuroscience 35, 1260-1273. 23. Wu, Z., Li, L., Xie, F., Du, J., Zuo, Y., Frost, J.A., Carlton, S.M., Walters, E.T. and Yang, Q. (2017). Activation of KCNQ Channels Suppresses Spontaneous Activity in Dorsal Root Ganglion Neurons and Reduces Chronic Pain after Spinal Cord Injury. Journal of neurotrauma. 24. Zheng, J.-H., Walters, E.T. and Song, X.-J. (2007). Dissociation of Dorsal Root Ganglion Neurons Induces Hyperexcitability That Is Maintained by Increased Responsiveness to cAMP and cGMP. Journal of neurophysiology 97, 15-25. 25. Davoody, L., Quiton, R.L., Lucas, J.M., Ji, Y., Keller, A. and Masri, R. (2011). Conditioned place preference reveals tonic pain in an animal model of central pain. The journal of pain : official journal of the American Pain Society 12, 868-874. 26. LaBuda, C.J. and Fuchs, P.N. (2000). A Behavioral Test Paradigm to Measure the Aversive Quality of Inflammatory and Neuropathic Pain in Rats. Experimental neurology 163, 490-494. 27. Sufka, K.J. (1994). Conditioned place preference paradigm: a novel approach for analgesic drug assessment against chronic pain. Pain 58, 355-366.

28. Sotocinal, S.G., Sorge, R.E., Zaloum, A., Tuttle, A.H., Martin, L.J., Wieskopf, J.S., Mapplebeck, Grimace Scale: a partially automated method for quantifying pain in the laboratory rat via facial expressions. Mol Pain. 7:55., 10.1186/1744-8069-1187-1155. place preference and aversion in mice. Nat Protoc 1, 1662-1670. running: a reliable and clinically relevant method to assess migraine pain in rats. The journal of headache and pain 18, 1-8.

31. Fuller, D.D., Lee, K.Z. and Tester, N.J. (2013). The impact of spinal cord injury on breathing during sleep. Respiratory physiology \& neurobiology 188, 344-354.

525 32. Jensen, M.P., Hirsh, A.T., Molton, I.R. and Bamer, A.M. (2010). Sleep Problems in Individuals 526 With Spinal Cord Injury: Frequency and Age Effects. Rehabilitation Psychology 54, 323-331. 
527 33. Altindag, O., Karagullu, H. and Gur, A. (2014). Orthopedics \& Muscular System : Current

528 Research Sleep Disturbances in Patients with Spinal Cord Injury. Orthopedics \& Muscular

529 System: Current Research 3, 10-13.

530 34. Castriotta, R., Wilde, M. and Sahay, S. (2012). Sleep Disorders in Spinal Cord Injury. Sleep

531 and Neurorehabilitation 7.

532 35. Lavela, S.L., Burns, S.P., Goldstein, B., Miskevics, S., Smith, B. and Weaver, F.M. (2012).

533 Dysfunctional sleep in persons with spinal cord injuries and disorders. Spinal cord 50, 682-685.

534 36. Norrbrink Budh, C., Hultling, C. and Lundeberg, T. (2005). Quality of sleep in individuals with

535 spinal cord injury: a comparison between patients with and without pain. Spinal cord 43, 85-95.

536 37. Sankari, A., Bascom, A., Oomman, S. and Badr, M.S. (2014). Sleep Disordered Breathing in

537 Chronic Spinal Cord Injury. Journal of Clinical Sleep Medicine 10.

538 38. Scheer, F., Zeitzer, J., Ayas, N., Brown, R., Czeisler, C. and Shea, S. (2006). Reduced sleep

539 efficiency in cervical spinal cord injury; association with abolished night time melatonin

540 secretion. Spinal cord 44, 78-81.

541 39. Klefbeck, B., Sternhag, M., Weinberg, J., Levi, R., Hultling, C. and Borg, J. (1998). Obstructive

542 sleep apneas in relation to severity of cervical spinal cord injury. Spinal cord 36, 621-628.

543 40. Noble, D.J., Martin, K.K., Parvin, S. and Garraway, S.M. (2018). Spontaneous and Stimulus-

544 Evoked Respiratory Rate Elevation Corresponds to Development of Allodynia in Spinal Cord-

545 Injured Rats. Journal of neurotrauma 36, 1909-1922.

546 41. Garraway, S., Watkins, K. and Hochman, S. (2015). (282) The development of mechanical

547 allodynia after spinal cord injury is associated with higher respiratory rate. The Journal of Pain

54816, S46.

549 42. Noble, D.J.M., C.J.; McKinnon, M.L.; Neblett, T.I.; Goolsby, W.N.; Hochman, S. (2017). Use of

550 electric field sensors for recording respiration, heart rate, and stereotyped motor behaviors in

551 the rodent home cage. Journal of neuroscience methods 277, 88-100.

552 43. Lee, K.Z., Huang, Y.J. and Tsai, I.L. (2014). Respiratory motor outputs following unilateral

553 midcervical spinal cord injury in the adult rat. Journal of applied physiology (Bethesda, Md. :

554 1985) 116, 395-405.

555 44. Kloefkorn, H., Aiani, L.M., Lakhani, A., Nagesh, S., Moss, A., Goolsby, W., Rehg, J.M.,

556 Pedersen, N.P. and Hochman, S. (2020). Noninvasive three-state sleep-wake staging in mice

557 using electric field sensors. Journal of neuroscience methods 344, 108834.

558 45. Kerr, B.J. and David, S. (2007). Pain behaviors after spinal cord contusion injury in two

559 commonly used mouse strains. Experimental neurology 206, 240-247.

560 46. Kloefkorn, H., Aiani, L., Lakhani, A., Nagesh, S., Moss, A., Goolsby, W., Rehg, J., Pedersen, N.

561 and Hochman, S. (2019). Noninvasive Sleep Scoring in Mice using Electric Field Sensors. bioRxiv, 562794552.

563 47. Chaplan, S.R., Bach, F.W., Pogrel, J.W., Chung, J.M. and Yaksh, T.L. (1994). Quantitative

564 assessment of tactile allodynia in the rat paw. Journal of neuroscience methods 53, 55-63.

565 48. Idlett, S., Halder, M., Zhang, T.H., Quevedo, J., Brill, N., Gu, W., Moffitt, M. and Hochman, S.

566 (2019). Assessment of axonal recruitment using model-guided preclinical spinal cord

567 stimulation in the ex vivo adult mouse spinal cord. Journal of neurophysiology 122, 1406-1420.

568 49. Castany, S., Gris, G., Vela, J.M., Verdu, E. and Boadas-Vaello, P. (2018). Critical role of sigma-

5691 receptors in central neuropathic pain-related behaviours after mild spinal cord injury in mice.

570 Scientific reports 8, 3873. 
571 50. Esteves, A.M., Mello, M.T., Squarcini, C.F., Lancellotti, C.L., Comparoni, A. and Tufik, S.

572 (2007). Sleep patterns over 15-day period in rats with spinal cord injury. Spinal cord 45, 360-

573366.

574 51. Walters, E.T. (2012). Nociceptors as chronic drivers of pain and hyperreflexia after spinal 575 cord injury: an adaptive-maladaptive hyperfunctional state hypothesis. Front Physiol 3, 309.

576 52. Lee, K.Z. and Kuo, H.C. (2017). Vagal Control of Breathing Pattern after Midcervical

577 Contusion in Rats. Journal of neurotrauma 34, 734-745.

578 53. Lee, K.-z., Huang, Y.-j. and Tsai, I.-I. (2018). Respiratory motor outputs following unilateral

579 midcervical spinal cord injury in the adult rat. Journal of applied physiology, 395-405.

580 54. Komnenov, D., Solarewicz, J.Z., Afzal, F., Nantwi, K.D., Kuhn, D.M. and Mateika, J.H. (2018).

581 Intermittent hypoxia promotes recovery of respiratory motor function in spinal cord-injured

582 mice depleted of serotonin in the central nervous system. Journal of applied physiology, 545-

583557.

584 55. Duraku, L.S., Hossaini, M., Hoendervangers, S., Falke, L.L., Kambiz, S., Mudera, V.C.,

585 Holstege, J.C., Walbeehm, E.T. and Ruigrok, T.J.H. (2012). Spatiotemporal Dynamics of Re-

586 Innervation and Hyperinnervation Patterns by Uninjured CGRP Fibers in the Rat Foot Sole

587 Epidermis after Nerve Injury. Molecular pain 8, 1744-8069-1748-1761.

588 56. Kleggetveit, I.P., Namer, B., Schmidt, R., Helas, T., Ruckel, M., Orstavik, K., Schmelz, M. and

589 Jorum, E. (2012). High spontaneous activity of C-nociceptors in painful polyneuropathy. Pain

590 153, 2040-2047.

591 57. Schmidt, R., Kleggetveit, I.P., Namer, B., Helas, T., Obreja, O., Schmelz, M. and Jorum, E.

592 (2012). Double spikes to single electrical stimulation correlates to spontaneous activity of

593 nociceptors in painful neuropathy patients. Pain 153, 391-398.

594 58. Serra, J., Bostock, H., Solà, R., Aleu, J., García, E., Cokic, B., Navarro, X. and Quiles, C. (2012).

595 Microneurographic identification of spontaneous activity in C-nociceptors in neuropathic pain

596 states in humans and rats. Pain 153, 42-55.

597 59. Serra, J., Solà, R., Aleu, J., Quiles, C., Navarro, X. and Bostock, H. (2011). Double and triple

598 spikes in C-nociceptors in neuropathic pain states: An additional peripheral mechanism of

599 hyperalgesia. PAIN ${ }^{\circledR} 152,343-353$.

600 